\title{
Single valproic acid treatment inhibits glycogen and RNA ribose turnover while disrupting glucose-derived cholesterol synthesis in liver as revealed by the $\left[\mathrm{U}_{-}{ }^{13} \mathrm{C}_{6}\right]-\mathrm{D}$-glucose tracer in mice
}

\author{
Richard D. Beger · Deborah K. Hansen · Laura K. Schnackenberg • \\ Brandie M. Cross · Javad J. Fatollahi · F. Tracy Lagunero • \\ Zoltan Sarnyai · Laszlo G. Boros
}

Received: 24 October 2008/Accepted: 4 March 2009/Published online: 31 March 2009

(C) The Author(s) 2009. This article is published with open access at Springerlink.com

\begin{abstract}
Previous genetic and proteomic studies identified altered activity of various enzymes such as those of fatty acid metabolism and glycogen synthesis after a single toxic dose of valproic acid (VPA) in rats. In this study, we demonstrate the effect of VPA on metabolite synthesis flux rates and the possible use of abnormal ${ }^{13} \mathrm{C}$ labeled glucosederived metabolites in plasma or urine as early markers of toxicity. Female CD-1 mice were injected subcutaneously with saline or $600 \mathrm{mg} / \mathrm{kg}$ ) VPA. Twelve hours later, the mice were injected with an intraperitoneal load of $1 \mathrm{~g} / \mathrm{kg}$ [U- ${ }^{13} \mathrm{C}$ ]-D-glucose. ${ }^{13} \mathrm{C}$ isotopomers of glycogen glucose and RNA ribose in liver, kidney and brain tissue, as well as glucose disposal via cholesterol and glucose in the plasma and urine were determined. The levels of all of the positional ${ }^{13} \mathrm{C}$ isotopomers of glucose were similar in plasma, suggesting that a single VPA dose does not disturb glucose
\end{abstract}

The views presented here do not necessarily reflect those of the U.S. Food and Drug Administration.

R. D. Beger $(\bowtie) \cdot$ D. K. Hansen · L. K. Schnackenberg National Center for Toxicological Research (NCTR), United States Food and Drug Administration, Jefferson, AR, USA e-mail: Richard.Beger@fda.hhs.gov

B. M. Cross - J. J. Fatollahi · F. T. Lagunero - L. G. Boros SiDMAP, LLC., Los Angeles, CA, USA

L. G. Boros

e-mail: lboros@sidmap.com

Z. Sarnyai

Department of Pharmacology, University of Cambridge, Cambridge, UK

L. G. Boros

UCLA School of Medicine, University of California,

Los Angeles, CA, USA absorption, uptake or hepatic glucose metabolism. Threehour urine samples showed an increase in the injected tracer indicating a decreased glucose re-absorption via kidney tubules. ${ }^{13} \mathrm{C}$ labeled glucose deposited as liver glycogen or as ribose of RNA were decreased by VPA treatment; incorporation of ${ }^{13} \mathrm{C}$ via acetyl-CoA into plasma cholesterol was significantly lower at $60 \mathrm{~min}$. The severe decreases in glucose-derived carbon flux into plasma and kidney-bound cholesterol, liver glycogen and RNA ribose synthesis, as well as decreased glucose re-absorption and an increased disposal via urine all serve as early flux markers of VPA-induced adverse metabolic effects in the host.

Keywords Valproic acid - Stable isotope-based dynamic metabolic profiling (SiDMAP) $\cdot\left[\mathrm{U}_{-}{ }^{13} \mathrm{C}_{6}\right]$-D-glucose

\section{Introduction}

Valproic acid (2-propyl-pentanoic acid, VPA) has clinical applications in the treatment of a variety of neuropsychiatric illnesses such as epilepsy and bipolar disorder (Simon and Penry 1975; Keane et al. 1982); however, it has been shown to act as a hepatotoxin in some patients (Eadie et al. 1988). While hepatotoxicity of VPA is not well-understood, numerous studies have identified several metabolites that are potentially responsible for the observed toxicity (Tang and Abbott 1996, 1997; Silva et al. 2004; Gopaul et al. 2000; 2003). Liver toxicity occurs with an overall incidence of 1 in 20,000, but a frequency as high as one in 600 or one in 800 is also common in high-risk groups such as infants below 2 years of age receiving anticonvulsant polytherapy (Perucca 2002). Early reports suggested that VPA toxicity might be due to decreased oxidative 
phosphorylation with uncoupling in isolated rat liver mitochondria. These effects are similar to those of propionate and isovalerate, suggesting a common mechanism of mitochondrial stress. This in turn may lead to the hepatocerebral disorder seen in Reye syndrome (Haas et al. 1981). In a multi-age rat model (10-, 25-, 40-, 80-day-old), increasing doses of VPA induced toxicity that was evident in all age groups after 4 days of treatment. However, toxicity patterns in the multi-age study were different within each age group. The most severe liver and spleen lesions were found mostly in 10 - or 80 -day-old rats. While significant changes in blood urea nitrogen occurred, altered alanine aminotransferase and alkaline phosphatase activities were observed in 10-day-old pups after treatment with even low doses of VPA (160 mg/kg). The highest VPA dose $(650 \mathrm{mg} / \mathrm{kg})$ caused significant decreases in the levels of serum total protein in older rats (40- and 80-day) (Espandiari et al. 2007).

An integrated metabonomics, proteomics and gene expression microarray systems biology study in CD-1 female pregnant mice was done after a single subcutaneous VPA dose of $600 \mathrm{mg} / \mathrm{kg}$ body weight (Schnackenberg et al. 2006). Urine, serum, and liver were examined at 6, 12, and $24 \mathrm{~h}$ after dosing and showed significantly increased glucose concentrations, glycogen phosphorylase activity and amylo-1,6-glucosidase protein levels. However, the mRNA levels of 20,000 liver genes did not reveal significantly altered expression at any of the time points examined. The elevated metabolic enzymes are involved in and control glucose metabolism to glycogen in liver and other mammalian tissues; therefore an early perturbation in the glycogenolysis process in the VPA exposed liver is evident (Schnackenberg et al. 2006).

In search of an early toxicity marker and in order to directly determine organ level metabolite flux and intermediate turnover rates, we used $\left[\mathrm{U}_{-}{ }^{13} \mathrm{C}_{6}\right]$-D-glucose as a tracer. We investigated hepatic glucose production, glucose dependent futile cycling, cholesterol synthesis, and glucose disposal via urine after a single VPA dose to female mice. We report that a single VPA dose in vivo significantly diminished the capacity of the liver to synthesize glycogen, ribose and cholesterol from glucose. We also observed urine glucose positional ${ }^{13} \mathrm{C}$ isotopomers consistent with increased glucose disposal and limited re-absorption from the primary filtrate. We therefore conclude that the previously reported decrease in plasma albumin and globulin levels after prolonged VPA treatment may be the result of the slow rate of messenger, transfer and ribosomal RNA backbone ribose synthesis. Decreased glycogen turnover and diminished cholesterol synthesis rather mimic a glycogen storage and mobilization type disease with functional changes in sterol synthesis as the earliest detectable mechanisms of valproate toxicity in the serum metabolome.

\section{Materials and methods}

\subsection{Animals}

Adult (8-9 weeks of age) female CD-1 strain mice (originally purchased from Charles River; Wilmington, MA, and maintained at the National Center for Toxicological Research; Jefferson, AR) were used for all experiments. Animals were housed in cages $(n=4)$ and maintained in a controlled environment $\left(22^{\circ} \mathrm{C}\right.$ with a 12 -h light-dark cycle). Mice had access to Purina rodent laboratory chow (Purina Mills, St. Louis, MO) and water ad libitum. Groups of 8 mice were injected subcutaneously with saline or $600 \mathrm{mg} / \mathrm{kg}$ VPA. Twelve hours later, they were injected with $1 \mathrm{~g} / \mathrm{kg}$ body weight [U- ${ }^{13} \mathrm{C}$ ]-D-glucose (Cambridge Isotope Laboratories Inc., Andover, MA, $>98 \%$ isotopic purity and positional accuracy) intraperitoneally. Tracer $\left({ }^{13} \mathrm{C}\right)$ carbon labeled glucose in plasma collected from the saphenous vein (30-50 $\mu \mathrm{l}$ ) was determined 60 and $120 \mathrm{~min}$ later. The mice were sacrificed $180 \mathrm{~min}$ after the glucose tracer load by over exposure to carbon dioxide. Terminal blood samples were collected by cardiac puncture, and liver, kidneys, whole brain (cortex) and urine were collected and frozen at $-80^{\circ} \mathrm{C}$. Samples were sent on dry ice overnight to SiDMAP, LLC and stored at $-80^{\circ} \mathrm{C}$ until analyzed using GC/MS-based mass isotopomer analysis. Stable ${ }^{13} \mathrm{C}$ labeled fractions and isotopomers of glucose in plasma and urine were determined according to a standard glucose tolerance test, which was broadened by glucose ${ }^{13} \mathrm{C}$ isotopomer analysis for determining glucose absorption, tissue uptake as well as known hepatic glucose production patterns. Incorporation of the ${ }^{13} \mathrm{C}$ tracer into glycogen, RNA ribose and cholesterol was also determined in brain, kidney and liver tissues. All animal care procedures followed those in the "National Research Council: Guide for the Care and Use of Laboratory Animals" (NRC, 1996 Washington, DC: Institute for Laboratory Animal Resources) and were approved by the Animal Care and Use Committee of the National Center for Toxicological Research.

\subsection{Stable Isotope Tracer Studies}

Glucose levels from $10 \mu \mathrm{l}$ plasma and urine were measured using ACCU-CHEK Advantage (Roche, Manheim, Germany). For mass isotopomer analysis, plasma or urine $(50 \mu \mathrm{l})$ samples were deproteinized using $0.1 \mathrm{ml} 0.3 \mathrm{~N}$ Barium Hydroxide and Zinc Sulfate solutions (SIGMA, St. Louis, MO), and the derived glucose fraction was treated with $1 \mathrm{ml}$ of $30 \mathrm{mg} / \mathrm{ml}$ hydroxylamine hydrochloride (Aldrich, St. Louis. MO) in pyridine (Aldrich, Milwaukee, WI) $\left(100^{\circ} \mathrm{C}\right.$ for $\left.1 \mathrm{~h}\right)$ and $0.1 \mathrm{ml}$ acetic anhydride (EMD, Gibbstown, NJ) $\left(100^{\circ} \mathrm{C}\right.$ for $\left.0.5 \mathrm{~h}\right)$. This created the aldonitrile pentaacetate derivative for GC/MS analysis (Katz 


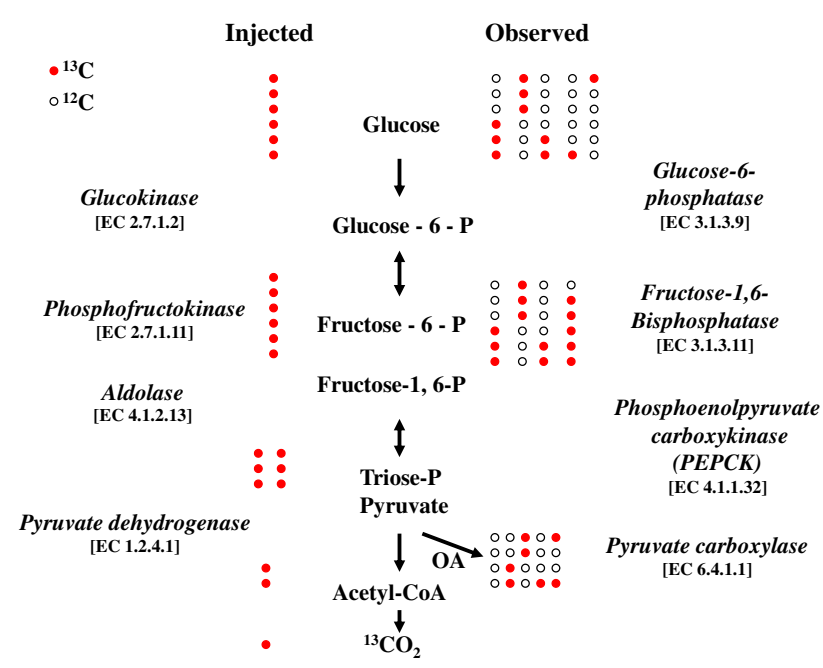

Fig. 1 Principles of mass isotopomer analysis (MIDA). The intraperitoneally injected $\left[\mathrm{U}^{13} \mathrm{C}_{6}\right.$ ]-D-glucose tracer [shown with all ${ }^{13} \mathrm{C}$ carbons with red filled circles] breaks down via enzymatic steps of glycolysis while via gluconeogenesis it loses, exchanges and dilutes its carbon skeleton with ${ }^{12} \mathrm{C}$ [shown with empty circles] before reappearing in plasma via hepatic glucose production or gets deposited into cellular glycogen in tissues. Various positional ${ }^{13} \mathrm{C}$ glucose isotopomers observed in plasma or glycogen depict specific reactions that contribute to gluconeogenesis, hepatic glucose production and glucose dependent futile cycles in the liver (plasma glucose) or tissues (glycogen glucose obtained from organs) $(\mathrm{OA}=$ oxaloacetate). (Color figure online)

et al. 1991). Glucose molecular ion and its positional isotopomers were monitored at the $m / z 328$ ion cluster for positional ${ }^{13} \mathrm{C}$ mass isotopomer distribution analyses (MIDA; Fig. 1) (Puchowicz et al. 1999; Xu et al. 2003).

Plasma cholesterol was extracted using $20 \mu \mathrm{l}$ blood samples, and tissue cholesterol was extracted by saponification of the Trizol (1.0 ml, Invitrogen, Carlsbad, CA) cell extract after removal of the upper glycogen and RNA containing supernatant using $30 \% \mathrm{KOH}$ and $100 \%$ ethanol (300 $\mu \mathrm{l} \mathrm{each)} \mathrm{for} 2 \mathrm{~h}$. Because Trizol contains both phenol (CAS No.: 108-95-2; 50\% by volume) and thiocyanate (CAS No.: $0022 ; 30 \%$ by volume), which may potentially interfere with fatty acid and cholesterol GC/MS analyses we also tested poor Trizol extracts using linear electron impact as well as cholesterol monitoring GC/MS runs after sterol extraction with no detectable sterol fragment impurities in Trizol solution only. Sterol extraction from tissues and plasma was performed using $5 \mathrm{ml}$ petroleum ether (EMD, Gibbstown, NJ) with repeated shaking for $20 \mathrm{~s}$ three times (Crick and Carroll 1987). The molecular ion of cholesterol was monitored at $\mathrm{m} / \mathrm{z} 386$ ion cluster. The enrichment of acetyl units in plasma and tissue cholesterol in response to VPA treatment was determined using the mass isotopomer distribution analysis (MIDA) approach. Cholesterol synthesis is dependent on glucose carbons since they are the primary source of acetyl-CoA, the carbon of which is then incorporated into fatty acids and cholesterol by de novo synthesis. Acetyl-CoA enrichment was calculated from the $m_{4} / m_{2}$ ratio using the formula $m_{4} / m_{2}=(n-1) / 2(p / q)$, where $n$ is the number of acetyl units, $p$ is the ${ }^{13} \mathrm{C}$ labeled precursor acetate fraction and $q$ is the ${ }^{12} \mathrm{C}$ labeled natural acetate fraction $(p+q=1)$ (Lee 1996).

RNA ribose and cellular glycogen were isolated by $2 \mathrm{~h}$ acid hydrolysis in $2 \mathrm{~N} \mathrm{HCl}$ of the cellular RNA chloroform-isopropanol fraction after Trizol-purification of cell extracts (Invitrogen, Carlsbad, CA). Ribose isolated from RNA, and glucose isolated from cellular glycogen (Powell et al. 2006) were derivatized to their aldonitrile acetate form using hydroxyl-amine in pyridine and acetic anhydride as described for plasma glucose above. We monitored the ion cluster around the $m / z 256$ (carbons $1-5$ of ribose, chemical ionization, CI), in order to find the molar enrichment and positional distribution of ${ }^{13} \mathrm{C}$ labeled carbons in total cellular messenger, ribosomal and transfer RNA-derived ribose (Lee et al. 1998).

\subsection{Gas chromatography/mass spectrometry (GC/MS)}

Mass spectral data were obtained on the Agilent 5975 Inert XL Mass Selective Detector connected to an HP6890N Network gas chromatograph. The settings are as follows: GC inlet $230^{\circ} \mathrm{C}$, transfer line $280^{\circ} \mathrm{C}$, MS source $230^{\circ} \mathrm{C}$, MS Quad $150^{\circ} \mathrm{C}$. An HP-5 capillary column $(30 \mathrm{~m}$ length, $250 \mu \mathrm{m}$ diameter, $0.25 \mu \mathrm{m}$ film thickness) was used for glucose (plasma, urine or glycogen) and RNA ribose analysis. A ZB-1 100\% polydimethylsiloxane (Phenomenex, Torrance, CA., USA) column (15 m length, $250 \mu \mathrm{m}$ diameter, $0.25 \mu \mathrm{m}$ film thickness) was used for cholesterol analysis with specific temperature programming for sterol recovery around $25 \mathrm{~min}$ retention time. ${ }^{13} \mathrm{C}$ positional enrichment is presented as ${ }^{13} \mathrm{C} \mathrm{M} 1, \mathrm{M} 2, \ldots, \mathrm{Mn}$, where $\mathrm{M}$ represents mass shift in Daltons (D) with an integer indicating the number of ${ }^{13} \mathrm{C}$ carbons replacing ${ }^{12} \mathrm{C}$ in metabolites. For example M2 in glucose indicates two ${ }^{13} \mathrm{C}$ exchanges with ${ }^{12} \mathrm{C}$ in glucose of plasma, urine or glycogen glucose as indicated in the text or legends.

\subsection{Data analysis and statistical methods}

In vivo experiments were carried out using eight mice for each treatment regimen. Mass spectral analyses were carried out by consecutive and independent automatic injections of $1 \mu \mathrm{l}$ sample by the automatic sampler; analyses were accepted only if the standard sample deviation was less than $1 \%$ of the normalized peak intensity among repeated injections. Data download was performed by three consecutive manual peak integrations using modified (background subtracted) spectra under the overlapping 
isotopomer peaks of the total ion chromatogram (TIC) window displayed by the Chemstation (Agilent, Palo Alto, CA) software. Statistical analyses were performed using the parametric unpaired heteroscedastic, two-tailed and one-tailed independent sample " $t$ " test as indicated with $95 \%$ confidence intervals, and $P<0.05$ was considered to indicate significant differences in glucose carbon metabolism in control or VPA $(600 \mathrm{mg} / \mathrm{kg})$ treated mice. Regression analysis was used to test the dependence (dependent or response variable) of cholesterol ${ }^{13} \mathrm{C}$ labeling and acetyl-CoA enrichment from plasma tracer $\left[\mathrm{U}_{-}{ }^{13} \mathrm{C}_{6}\right]$-D-glucose as the independent variable (explanatory variables). Cholesterol ${ }^{13} \mathrm{C}$ labeling and acetyl-CoA enrichment from the plasma glucose tracer in the regression equation was modeled as the corresponding constants random variable representing unexplained variation in the dependent variable as the null hypothesis. Regression statistics, analysis of variance (ANOVA) and linear regression were generated using the best fit model with the least squares as the primary criteria of VPA toxicity after a single dose treatment (Lindley 1987).

\section{Results}

We utilized the mass isotopomer distribution analysis MIDA approach in order to learn about single dose plasma, urine or tissue toxicity mechanisms and markers in VPAtreated mice using a state of the art metabolic tracer technology and the uniformly ${ }^{13} \mathrm{C}$ labeled $\left[\mathrm{U}_{-}{ }^{13} \mathrm{C}_{6}\right]$-D-glucose tracer. Figure 1 shows the concept of the MIDA approach and the major mass isotopomers generated via futile cycles by the liver or tissues.

Plasma glucose levels were similar in control and dosed animals at all time points, namely $181 \mathrm{mg} / \mathrm{dl}(\mathrm{SD}=10)$ at $0 \mathrm{~min}, 319 \mathrm{mg} / \mathrm{dl}(\mathrm{SD}=9)$ at $60 \mathrm{~min}, 278 \mathrm{mg} / \mathrm{dl}(\mathrm{SD}=$ $11.96)$ at $120 \mathrm{~min}$ and $217 \mathrm{mg} / \mathrm{dl}(\mathrm{SD}=14)$ at $180 \mathrm{~min}$ in control, and $186 \mathrm{mg} / \mathrm{dl}(\mathrm{SD}=13)$ at $0 \mathrm{~min}, 322 \mathrm{mg} / \mathrm{dl}$ $(\mathrm{SD}=7)$ at $60 \mathrm{~min}, 277 \mathrm{mg} / \mathrm{dl}(\mathrm{SD}=10)$ at $120 \mathrm{~min}$ and $221 \mathrm{mg} / \mathrm{dl}(\mathrm{SD}=21)$ at $180 \mathrm{~min}$ in VPA treated animals, respectively. On the other hand, 180 min collected urine glucose content was increased from $4.9 \mathrm{mg} / \mathrm{dl}(\mathrm{SD}=1.6$; $n=4)$ in control mice to $32 \mathrm{mg} / \mathrm{dl}(\mathrm{SD}=9.2 ; n=6$; $P<0.001)$ in VPA-treated animals. Plasma ${ }^{13} \mathrm{C}$-glucose labeled fractions including all isotopomers of ${ }^{13} \mathrm{C}$-labeled glucose showed almost identical enrichment in control and VPA treated animals. The ${ }^{13} \mathrm{C}$ labeled fractions in plasma glucose were $50.9 \%, 24.9 \%$ and $13.2 \%$ at 60,120 and $180 \mathrm{~min}$, respectively, after saline; or $51.9 \%, 26.7 \%$ and $15.3 \%$ after VPA treatment as shown in Fig. 2. This indicates that a single VPA dose did not alter tracer glucose uptake from the intraperitoneal cavity or clearance from the plasma by host organs.

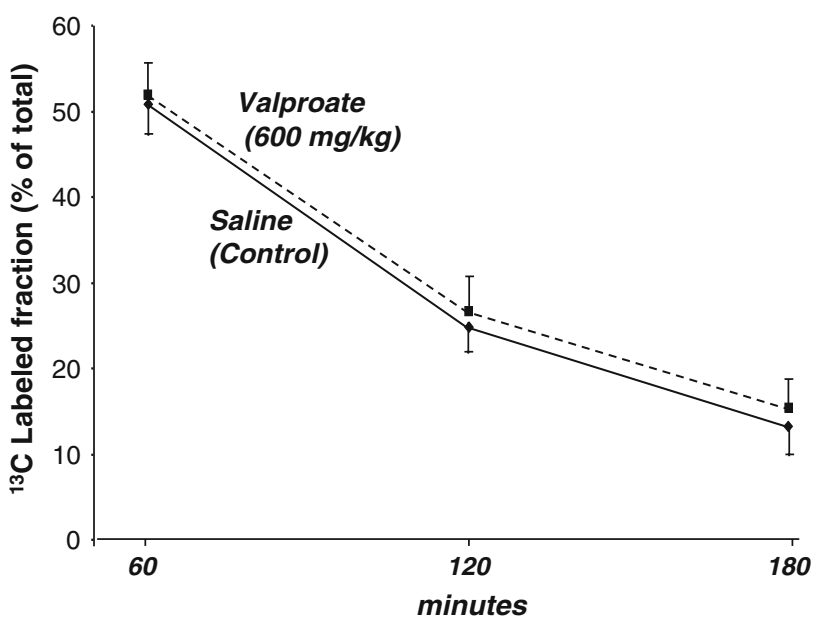

Fig. 2 Intraperitoneal ${ }^{13} \mathrm{C}$ tracer glucose tolerance test (IPGTT). Glucose ${ }^{13} \mathrm{C}$ labeled fractions as per cent of total glucose in plasma (Y axis, $\left.\Sigma \mathrm{m}_{\mathrm{n}=\mathrm{C} 1-\mathrm{C} 6}\right)$ which includes all ${ }^{13} \mathrm{C}$-labeled positional isotopomers of glucose at $0,60,120$ and $180 \mathrm{~min}$ (X axis) after tracer load. Control and VPA-treated animals show virtually identical ${ }^{13} \mathrm{C}$ labeled glucose fractions in plasma indicating similar absorption and clearance of tracer glucose at all time points. Control (lower continuous line) or VPA (upper broken line) glucose ${ }^{13} \mathrm{C}$-labeled plasma fractions as independent variables are compared using regression analysis to corresponding plasma cholesterol ${ }^{13} \mathrm{C}$-labeled fractions as the dependent variable to determine glucose to cholesterol flux by liver metabolism in Tables 1 and 2 (mean + standard deviation (SD); $n=8$ )

In the ${ }^{13} \mathrm{C}$ labeled plasma glucose fraction, the injected [U- ${ }^{13} \mathrm{C}_{6}$ ]-D-glucose tracer accounted for $63.1 \%, 29.4 \%$ and $12.9 \%$ of total label in saline-treated animals or $61.9 \%$, $25.1 \%$ and $12.7 \%$ after VPA treatment at 60,120 and $180 \mathrm{~min}$, respectively. Various isotopomers of plasma glucose are shown in Fig. 3 indicating similar glucose absorption and disposal patterns in plasma via hepatic glucose production and futile cycling in the liver. Control and VPA treated animals show virtually identical ${ }^{13} \mathrm{C}$ labeled glucose isotopomer fractions in plasma indicating similar absorption (M6, glucose tracer injected at time 0), as well as hepatic glucose production and futile cycles in liver at all time points.

Although plasma glucose ${ }^{13} \mathrm{C}$ labeling patterns did not show significant alterations in response to a single VPA dose, plasma cholesterol ${ }^{13} \mathrm{C}$ acetyl-CoA enrichment at $60 \mathrm{~min}$ after glucose administration was severely decreased in dosed animals. Glucose-derived acetate enrichment of plasma cholesterol was $10.93 \%, 5.83 \%$ and $3.19 \%$ at 60,120 and $180 \mathrm{~min}$, respectively, after saline; or $6.52 \%, 7.76 \%$ and $2.82 \%$ after $600 \mathrm{mg} / \mathrm{kg}$ VPA treatment as shown in Fig. 4. The summary output of regression statistics between ${ }^{13} \mathrm{C}$ labeled acetate enrichment in cholesterol from glucose is shown in Table 1 (control) and Table 2 (VPA treatment). Such regression statistics indicate that a single VPA dose severely decreases glucose-derived acetate flux towards 


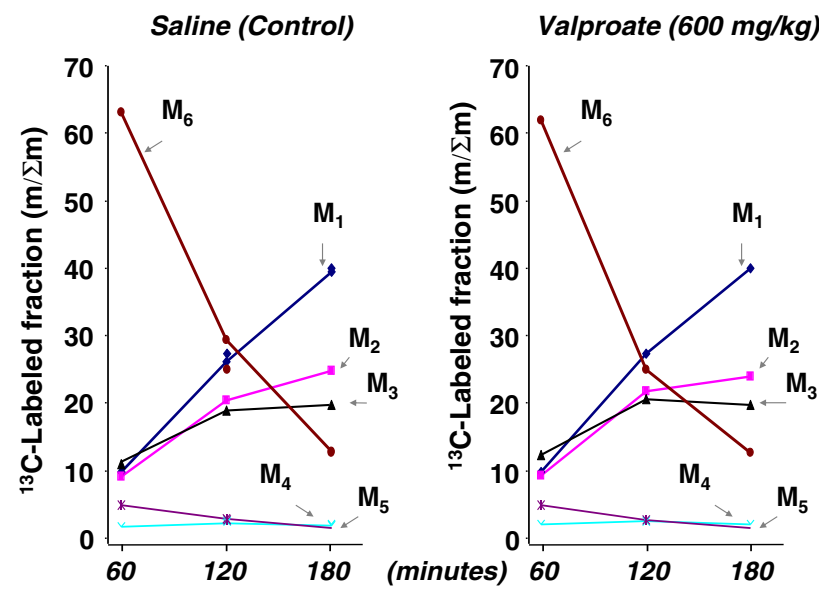

Fig. 3 All ${ }^{13} \mathrm{C}$ labeled glucose isotopomers (including the $\left[\mathrm{U}-{ }^{13} \mathrm{C}\right]-\mathrm{D}-$ glucose tracer) in plasma. Positional glucose ${ }^{13} \mathrm{C}$ isotopomer fractions as per cent of the total ${ }^{13} \mathrm{C}$ enriched fraction in plasma ( $\mathrm{Y}$ axis, $\left.\Sigma \mathrm{m}_{\mathrm{n}=\mathrm{C} 1,2,3,4,5,6}\right)$ at $0,60,120$ and $180 \mathrm{~min}$ (X axis) after tracer load. The various isotopomers indicate: M1, pyruvate carboxylase activity; M2, pyruvate dehydrogenase activity and complete TCA cycle turnaround; $\mathrm{M} 3$, glucose generated from $\left[\mathrm{U}_{-}{ }^{13} \mathrm{C}_{3}\right]$-DL-lactate and the Cori cycle; M4, non-oxidative pentose cycle; and M5, oxidative pentose cycle (mean; $n=8$ ) (Lee et al. 1991)

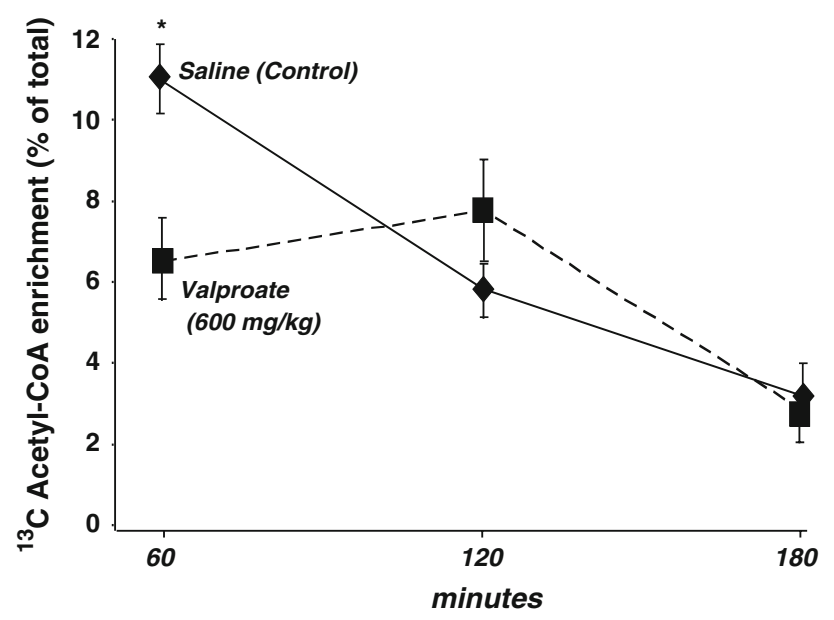

Fig. 4 Acetyl-CoA saturation curves of plasma cholesterol from the $\left[\mathrm{U}-{ }^{13} \mathrm{C}\right]-\mathrm{D}$-glucose tracer. Please also see Table 1 (control vehicle treatment) and Table 2 (VPA treatment) regression statistics (mean \pm standard deviation (SD); $n=8$ )

cholesterol synthesis as shown by the severely decreased $R^{2}$, $P$ value and control coefficient (Multiple $R$ ), after VPA treatment of the glucose-cholesterol regression curves.

It is particularly interesting that liver and brain tissuebound cholesterol remained relatively low-labeled with ${ }^{13} \mathrm{C}$ from the glucose tracer, namely $1.49 \%(\mathrm{SD}=0.114)$ and $1.41 \%(\mathrm{SD}=0.1238)$ in control, or $1.46 \%(\mathrm{SD}=0.1394$; NS) and $1.47 \%(\mathrm{SD}=0.1266$; NS) after VPA treatment. On the other hand, kidneys of control animals exhibited a $\sim 2.6$-fold higher $3.91 \%$ ( $\mathrm{SD}=0.2061){ }^{13} \mathrm{C}$ labeling in cholesterol, which was decreased to $3.64 \%$ ( $\mathrm{SD}=0.3953$;
Table 1 Regression statistics (A), analysis of variance (B) and linear regression (C) between ${ }^{13} \mathrm{C}$ glucose and glucose-derived acetate enrichment in cholesterol using the corresponding 60,120 and 180 minutes average plasma ${ }^{13} \mathrm{C}$ acetyl-CoA enrichment values in control vehicle treated animals

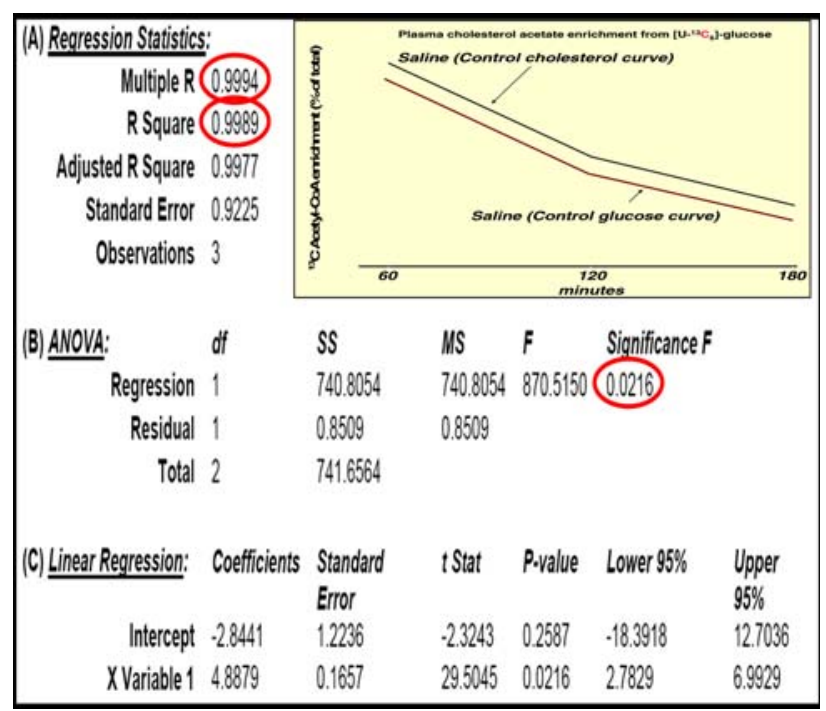

Table 2 Regression statistics (A), analysis of variance (B) and linear regression (C) between ${ }^{13} \mathrm{C}$ glucose and glucose-derived acetate enrichment in cholesterol using the corresponding 60, 120 and 180 minutes average plasma ${ }^{13} \mathrm{C}$ acetyl-CoA enrichment values in $V P A$ treated animals

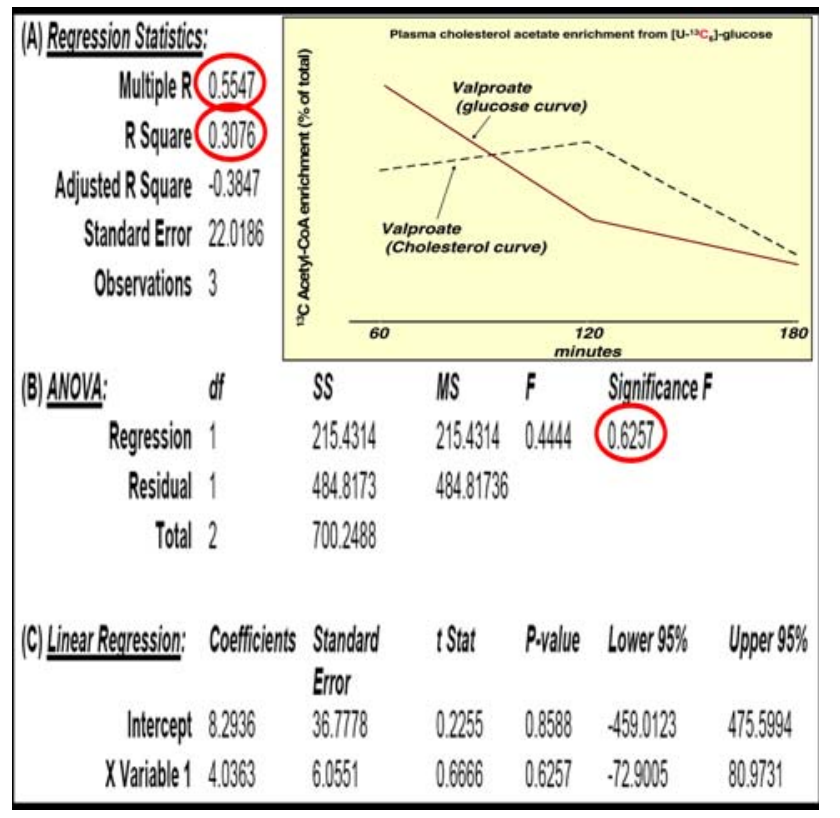

$* P=0.01514)$, consistent with a 7\% decrease after a single VPA treatment.

Liver glycogen (Fig. 5a) and RNA ribose (Fig. 5b) turnover rates were significantly decreased after VPA treatment in comparison with vehicle treated animals. The [U- ${ }^{13} \mathrm{C}_{6}$ ]-D-glucose tracer labeled $22.5 \%$ and $13.0 \%$ of 

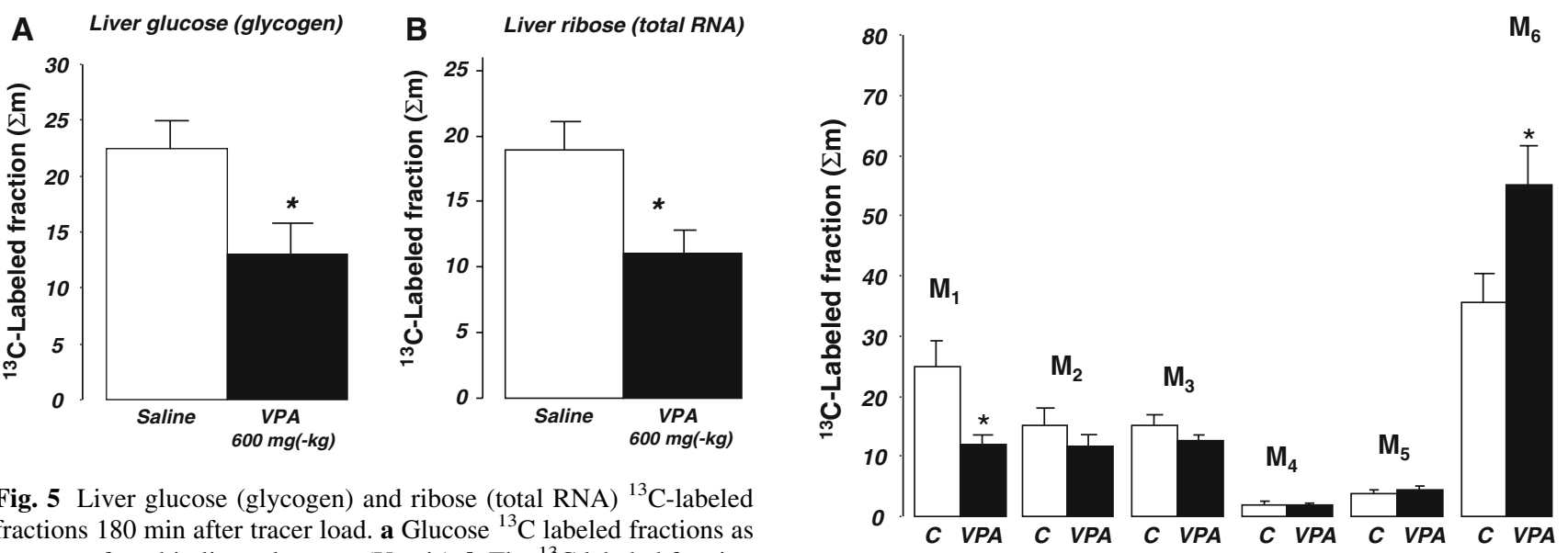

Fig. 5 Liver glucose (glycogen) and ribose (total RNA) ${ }^{13} \mathrm{C}$-labeled fractions $180 \mathrm{~min}$ after tracer load. a Glucose ${ }^{13} \mathrm{C}$ labeled fractions as per cent of total in liver glycogen ( $\mathrm{Y}$ axis), $\mathbf{b}$ The ${ }^{13} \mathrm{C}$ labeled fraction in RNA ribose (mean + standard error of the mean $(\mathrm{SEM}) ; n=8$ )

liver glycogen; and $19.0 \%$ and $11.0 \%$ of RNA ribose after saline and VPA treatment, respectively, during the $180 \mathrm{~min}$ tracer incubation period. In the brains of saline-treated mice only $1.39 \%(\mathrm{SD}=0.047)$ of RNA ribose was labeled with ${ }^{13} \mathrm{C}$ indicating a slower tissue specific RNA turnover compared to that of the liver. One single VPA dose further reduced brain-specific RNA ${ }^{13} \mathrm{C}$ labeling to $1.15 \%$ $(\mathrm{SD}=0.26)$, which closely approached significance by a " $P$ " value of 0.072 using the more severe two-tailed " $t$ " test. On the other hand, kidney RNA ribose exhibited $1.32 \%(\mathrm{SD}=0.16)$ and $1.41 \%(\mathrm{SD}=0.45 ; \mathrm{NS}){ }^{13} \mathrm{C}$ labeled fractions in control and VPA-treated mice, respectively. These data suggest a deceleration in the turnover rate of liver and brain but not the kidneys' RNA synthesis after single VPA dosing.

Three-hour aspirated urine samples from the bladder showed that, of the glucose found in the same amounts $(10 \mu \mathrm{l})$ of urine, $35.7 \%$ and $55.3 \%$ were the injected ${ }^{13} \mathrm{C}$ glucose tracer (Fig. 6) in control and VPA treated animals, respectively. The tracer labeled glucose fractions on one or two carbon positions were much less abundant in the urine, indicating a significant divergence between plasma and urine glucose isotopomers after VPA treatment. This suggests impaired glucose re-absorption from the primary glomerular filtrate by the proximal and distal tubules' glucose transport mechanisms.

\section{Discussion}

This report describes the fate of the $\left[\mathrm{U}_{-}{ }^{13} \mathrm{C}_{6}\right]$-D-glucose stable isotope substrate tracer in plasma, urine, liver, brain and kidneys of control and VPA treated mice. We have used a targeted substrate-product selection approach marking glucose-derived glycogen, nucleic acid and cholesterol synthesis and turnover rates, as well as hepatic glucose

Fig. 6 All ${ }^{13} \mathrm{C}$ labeled glucose isotopomers in aspirated urine from bladder $3 \mathrm{~h}$ after ${ }^{13} \mathrm{C}$ tracer glucose load. Glucose ${ }^{13} \mathrm{C}$ isotopomer fractions are shown as per cent of the total ${ }^{13} \mathrm{C}$ enriched fraction in urine (Y axis, $\Sigma \mathrm{m}_{\mathrm{n}=\mathrm{C} 1,2,3,4,5,6}$ ) in control (C) and valproic acid treated (VPA) animals (X axis). Control and VPA treated animals showed distorted ${ }^{13} \mathrm{C}$ labeled glucose isotopomer fractions in urine indicating increased tracer disposal via urine (M6, [U- $\left.{ }^{13} \mathrm{C}_{6}\right]$-D-glucose tracer injected at time 0 ; average $+\mathrm{SEM}, n=8 ; * P=0.016)$ in VPA treated animals. The various isotopomers indicate: M1, pyruvate carboxylase activity $(* P=0.029) ; \mathrm{M} 2$, pyruvate dehydrogenase activity and complete TCA cycle turnaround; M3, glucose generated from $\left[\mathrm{U}_{-}{ }^{13} \mathrm{C}_{3}\right]$-DL-lactate and the Cori cycle; M4, non-oxidative pentose cycle; and M5, oxidative pentose cycle

production and glucose dependent futile cycling as potential early functional metabolic biomarkers of VPA-induced toxicity. The non-invasive and non-radiating ${ }^{13} \mathrm{C}$ glucose tracer (Buchanan et al. 1998; Garg et al. 2005) allows realtime quantification of synthesis and turnover of selected metabolites in order to develop the necessary translational plasma, tissue and urine isotopomer profiles routinely applicable as toxicity biomarkers in humans. Furthermore, the positional stable ${ }^{13} \mathrm{C}$ isotope-based dynamic metabolic profiling approach has been shown to be valid and to provide additional information regarding glucose fluxes in comparison with more traditional metabolic approaches such as in hereditary hemochromatosis-related abnormalities that lead to cirrhosis, diabetes and other morbidities (Huang et al. 2007). Changes in vital metabolite fluxes in response to a single toxic dose of VPA may help in the determination of the mechanism of toxicity before structural or morphological damage develops. In short, our tracer substrate approach utilizes the accurate assessment and quantification of cross-talk and flux alterations among physiologically vital substrate and product pools and their disruption by valproate to provide early indications of the presence and mechanisms of valproate toxicity.

Presently VPA and other drug toxicities, in general, are assessed by morphological markers of organ damage including hepatotoxicity and microvesicular steatosis, proteomic and metabonomic methods (Schnackenberg et al. 
2006) and gene expression profiles (Lee et al. 2007). In the study by Lee et al. (2007), after microarray analysis of 1910 genes and hierarchical clustering, it was evident that gene expression data show striking changes in the synthesis of enzyme proteins associated with fatty acid and steroid metabolism. These authors used two doses of VPA (100 and $1000 \mathrm{mg} / \mathrm{kg}$ ) and examined gene expression at several time points. They observed changes in gene expression that were dependent both on VPA dose as well as time after treatment. Some of the genes responded soon after treatment (early genes), while others responded only at the later time points. VPA treatment characteristically up- or down-regulated (cutoff $>1.5$-fold) 60 genes involved in lipid metabolism and biological pathways for biosynthesis of triglycerides and cholesterol, catabolism of fatty acids, and lipid transport. One of the first reports to match a hepatotoxic VPA dose (200 or $600 \mathrm{mg}$ per kg, i.p.) with its functional and structural changes in rats revealed an immediate, dosedependent and prolonged increase in bile salt-independent bile flow with a decrease in biliary cholesterol and phospholipid output. At 3 and 5 h, marked structural changes were evident in hepatocytes, including formation of autophagic vacuoles, engulfing morphologically altered mitochondria and occasional peroxisomes (Jezequel et al. 1984). In our study VPA-treated liver exhibited decreased acetyl-CoA ${ }^{13} \mathrm{C}$ enrichment from iglucose to cholesterol by more than $40 \%$ in easily accessible plasma samples only $12 \mathrm{~h}$ after a single VPA dose, indicating a non-glucose responsive liver after a single dose of $600 \mathrm{mg}(-\mathrm{kg})$ VPA. This rapid toxicity marker is based and herein demonstrated to be applicable in vivo on the finding that a single VPA treatment already decreases the rate constant (Sarwal et al. 1989; Comi and Hamilton 1994) of glucose-derived ${ }^{13} \mathrm{C}$-labeled acetate's flux towards sterol synthesis.

An important functional impairment of liver cells is evident by the significantly decreased glycogen turnover with a concomitant and comparable decrease in RNA ribose synthesis. In a previous report, VPA at $200 \mathrm{mg} / \mathrm{kg}$ caused a profound reduction in liver glycogen stores in infant mice (Thurston et al. 1981), which is consistent with our previous results of a decrease in liver glucose after VPA administration (Schnackenberg et al. 2006). Our present results showed a more than $40 \%$ decrease in glycogen ${ }^{13} \mathrm{C}$ labeling from plasma tracer glucose after an intraperitoneal tracer load. This decreased glycogen turnover points to one of the central mechanisms of VPA-induced hepatotoxicity, namely the decrease in glucose storage capacity in the satiety state. Although plasma glucose ${ }^{13} \mathrm{C}$ isotopomer fractions did not yet reveal impaired hepatic glucose production or glucose dependent futile cycling, we believe that repeated treatments with VPA and a more prolonged depletion of liver glycogen could in fact impair gluconeogenesis and blood glucose control.
The liver is responsible for the production of the majority of plasma albumin and globulin, as well as various specialized proteins in host defense (such as complement cascade globulins) and of the extrinsic pathway of blood coagulation. In fact, delayed synthesis and activation of factors II, V, VII, X and fibrinogen are important measures of liver damage. Additionally, the significant decrease in liver and, less extensively, brain RNA ribose synthesis by VPA is another key mechanism of organ damage and available as an early signal of organ toxicity. Decreased RNA turnover may have far reaching and severe functional consequences affecting gene expression, protein synthesis and metabolism. Consequent abrupt decreases in protein synthesis and turnover can now sensitively be measured by deuterium labeled water as the tracer and matrix assisted time of flight (MALDI-TOF) technology for a more accurate determination of drug-interrupted liver cell protein synthesis of specific classes and function (Xiao et al. 2008). Functional protein analysis is a method that will likely increase the power of drug toxicity studies in the near future.

Our careful analysis of urine glucose ${ }^{13} \mathrm{C}$ isotopomers provides another easy-to-obtain biomarker for VPA toxicity in the kidney. The plasma distribution of glucose ${ }^{13} \mathrm{C}$ isotopomers is virtually identical in control and VPA-treated mice and follows the logical progression of higher $\left[\mathrm{U}^{13} \mathrm{C}_{6}\right]$ D-glucose tracer $60 \mathrm{~min}$ after dosing, with increases in the isotopomers of gluconeogenesis at 120 and $180 \mathrm{~min}$. Three hours after glucose dosing, the injected tracer is still the highest in the urine of the VPA group, while in controls much less of the tracer was recovered. These findings strongly suggest that VPA inhibits glucose re-absorption by the proximal tubules of the kidneys (Nowak and Schnellmann 1995; Cohen and Little 1976), resulting in a strikingly different urine isotopomer profile $3 \mathrm{~h}$ after glucose tracer load. While inhibited gluconeogenesis and glucose production by VPA in proximal tubules may be the cause of the uniformly decreased ${ }^{13} \mathrm{C}$ M1, M2 and M3 fractions in urine, the high fraction of the injected tracer (M6 fraction) more likely indicates decreased re-absorption of glucose from the primary filtrate in the proximal tubules.

The anticonvulsant activity of VPA may be due, in part, to alterations in brain glucose metabolism. It has previously been shown that VPA interferes with brain glucose transport and metabolism by inhibiting glucose transporter-1 (GLUT1) activity in normal and GLUT1-deficient erythrocytes by $20-30 \%$. This causes a corresponding reduction in $\mathrm{V}_{\max }$ of glucose transport in primary astrocytes as well as in normal and GLUT1-deficient fibroblasts. VPA inhibited glucose transport by $20-40 \%$, and this was accompanied by an up to $60 \%$ down regulation of GLUT1 mRNA expression (Wong et al. 2005). Additionally, fructose-1,6-bisphosphate (F1,6BP) shifts the metabolism of glucose from glycolysis to the pentose phosphate pathway 
and exerts anticonvulsant activity in several rat models of acute seizures induced by pilocarpine, kainic acid, or pentylenetetrazole. This is similar to the action of 2-deoxyglucose (2-DG; an inhibitor of glucose uptake and glycolysis) and VPA (Lian et al. 2007), which not only alter brain function but also vastly affect the disposal of glucose via urine, based on our tracer substrate-based metabolomics study.

It is also of particular physiological and toxicological importance that the kidneys are primary organs of HDLcholesterol storage, catabolism and disposal, based on the over 2.5 -fold increase in ${ }^{13} \mathrm{C}$ labeled cholesterol in the kidneys in comparison with liver and brain tissues, as well as by showing similar response to VPA induced changes in plasma cholesterol, as shown in this 3-h tracer substrate study. It has long been known that patients homozygous for Tangier disease (a familial high-density lipoprotein deficiency) have a near absence due to selective catabolism of plasma HDL apoA-I by the kidneys (Lee et al. 2005). This is the result of mutations in the ATP-binding cassette, subfamily $\mathrm{A}$, member 1 (ABCA1), which is a prominent member of the HDL assembly membrane transporters (Slatter et al. 2008). With cholesterol as its substrate, this protein functions as a lipid efflux pump in the cellular cholesterol removal pathway. It is obvious based on our study that the selective catabolism of circulating HDL in the kidneys and its ${ }^{13} \mathrm{C}$ cholesterol moiety leave a prominent stable isotope fingerprint on the kidneys' cholesterol ${ }^{13} \mathrm{C}$ silhouette, which opens new avenues to study glucose $\rightarrow$ cholesterol carbon exchange, disposal and substrate product cross-talk in future in vivo drug toxicity, efficacy and mechanism of action studies.

Renal tubular glucose re-absorption is performed chiefly by GLUT1 in the basolateral membrane in the proximal tubules in rats and humans (Linden et al. 2006). The finding in the current study of decreased re-absorption of glucose from the proximal tubules might be due to a decrease in kidney GLUT1 expression and activity. This decreased expression might also occur in the brain and might be the mechanism whereby valproate inhibits glucose transport in the brain for the effective control of seizures (Cornford and Oldendorf 1986). The ${ }^{13} \mathrm{C}$ glucose positional isotopomer profile of urine may serve as the most effective, least invasive and easiest to analyze biomarker to determine the whole body response to drugs targeting glucose transport.

\subsection{Concluding remarks}

The pharmaceutical industry is challenged to find biomarkers for early drug toxicity while attempting to achieve maximum drug efficacy to control various human diseases. We showed that VPA produces liver toxicity by disrupting flux of tracer glucose-derived acetate and its disposal via plasma cholesterol. The decreased re-absorption of glucose from the proximal tubules may indicate decreased glucose transport in the brain which may control seizures and psychiatric mental swings in bipolar disease. ${ }^{13} \mathrm{C}$ tracer substrate-based metabolomics may improve toxicology by uncovering defective metabolite fluxes on the basis of decreased correlation between tracer substrate flow into plasma products requiring liver passage and metabolism. As toxic drugs decrease rate constants and thus regression coefficients, select metabolic tracer substrates and their products may be used to depict and predict how gene expression profiles translate through metabolic interactions into the functional consequences of organ and biological systems activity, thereby avoiding iatrogenic harm and choosing optimal medical interventions in the clinic. Cutoff regression coefficients based on known toxic drug induced regression parameters need to be determined. Additionally, tracer methodology could be useful to monitor individual responses to drugs and to detect early signs of toxicity prior to irreversible organ damage or loss of life.

Acknowledgments We thank Tamas F. Boros for coordinating metabolite, enzyme convention (EC) names, and numbers for human enzyme isoforms with the International KEGG and BRENDA databases, Dale Chenoweth for final edit of the manuscript, and Gene White, Carrie Moland and Bionetic Animal Care Staff for work during this animal study. Financial or Material Support: All sources of funding for these studies have been drawn internally within the participating institutions as a service to governments of the United States and the United Kingdom in order to cover base salaries and material costs. More specifically, this work was performed pursuant to a Material Transfer Agreement by and between SiDMAP, LLC and the National Institutes of Health, the Food and Drug Administration, and the Centers for Disease Control and Prevention. No funds were transferred between Institutions for this work.

Conflict of interest We declare no conflict of interest in performing, presenting, discussing and concluding stable isotope-labeled biomarker metabolite profiles reported in this manuscript. None of the authors are or have been involved in the production or sales of the tracer glucose substrate, valproic acid, any chemical or product used to perform these studies. Furthermore the reported studies have been performed as a collaborative research agreement with no funds transferred between the institutions participating in the experiments and the results are aimed at improving drug toxicology assessments for the benefit of the Public.

Open Access This article is distributed under the terms of the Creative Commons Attribution Noncommercial License which permits any noncommercial use, distribution, and reproduction in any medium, provided the original author(s) and source are credited.

\section{References}

Buchanan, T. A., Xiang, A., Kjos, S. L., et al. (1998). Gestational diabetes: Antepartum characteristics that predict postpartum glucose intolerance and type 2 diabetes in Latino women. Diabetes, 47, 1302-1310. doi:10.2337/diabetes.47.8.1302. 
Cohen, J. J., \& Little, J. R. (1976). Lactate metabolism in the isolated perfused rat kidney: Relations to renal function and gluconeogenesis. The Journal of Physiology, 255, 399-414.

Comi, R. J., \& Hamilton, H. (1994). Reduction of red cell glucose transporter intrinsic activity in diabetes running. Hormone and Metabolic Research. Hormon- und Stoffwechselforschung. Hormones et Metabolisme, 26, 26-32. doi:10.1055/s-2007-1000767.

Cornford, E. M., \& Oldendorf, W. H. (1986). Epilepsy and the bloodbrain barrier. Advances in Neurology, 44, 787-812.

Crick, D. C., \& Carroll, K. K. (1987). Extraction and quantitation of total cholesterol, dolichol and dolichyl phosphate from mammalian liver. Lipids, 22, 1045-1048. doi:10.1007/BF02536448.

Eadie, M. J., Hooper, W. D., \& Dickinson, R. G. (1988). Valproateassociated hepatotoxicity and its biochemical mechanisms. Medical Toxicology, 3(2), 85-106.

Espandiari, P., Zhang, J., Schnackenberg, L. K., et al. (2007). Agerelated differences in susceptibility to toxic effects of valproic acid in rats. Journal of Applied Toxicology, (Nov), 9. Epub ahead of print.

Garg, M., Bassilian, S., Bell, C., Lee, S., \& Lee, W. N. (2005). Hepatic de novo lipogenesis in stable low-birth-weight infants during exclusive breast milk feedings and during parenteral nutrition. JPEN, Journal of Parenteral and Enteral Nutrition, 29, 81-86. doi:10.1177/014860710502900281.

cGopaul, S., Farrell, K., \& Abbott, F. (2000). Gas chromatography/ negative ion chemical ionization mass spectrometry and liquid chromatography/electrospray ionization tandem mass spectrometry quantitative profiling of $\mathrm{N}$-acetylcysteine conjugates of valproic acid in urine: Application in drug metabolism studies in humans. Journal of Mass Spectrometry, 35, 698-704. doi:10.1002/ 1096-9888(200006)35:6<698::AID-JMS996>3.0.CO;2-S

Gopaul, S., Farrell, K., \& Abbott, F. (2003). Effects of age and polytherapy, risk factors of valproic acid (VPA) hepatotoxicity, on the excretion of thiol conjugates of (E)-2,4-diene VPA in people with epilepsy taking VPA. Epilepsia, 44, 322-328. doi: 10.1046/j.1528-1157.2003.07202.x.

Haas, R., Stumpf, D. A., Parks, J. K., \& Eguren, L. (1981). Inhibitory effects of sodium valproate on oxidative phosphorylation. Neurology, 31, 1473-1476.

Huang, J., Gabrielsen, J. S., Cooksey, R. C., et al. (2007). Increased glucose disposal and AMP-dependent kinase signaling in a mouse model of hemochromatosis. The Journal of Biological Chemistry, (Oct), 30. Epub ahead of print.

Jezequel, A. M., Bonazzi, P., Novelli, G., Venturini, C., \& Orlandi, F. (1984). Early structural and functional changes in liver of rats treated with a single dose of valproic acid. Hepatology (Baltimore Md), 4, 1159-1166. doi:10.1002/hep.1840040611.

Katz, J., Wals, P. A., \& Lee, W. N. (1991). Determination of pathways of glycogen synthesis and the dilution of the three-carbon pool with $\left[\mathrm{U}-{ }^{13} \mathrm{C}\right]$ glucose. Proceedings of the National Academy of Sciences of the United States of America, 88, 2103-2107. doi:10.1073/pnas.88.6.2103.

Keane, P. E., Meldrum, B. S., Simiand, J., \& Vernieres, J. C. (1982). Mechanism of anticonvulsant action of valproate. Progress in Neurobiology, 19, 315-359. doi:10.1016/0301-0082(82)90010-7.

Lee, W. N. (1996). Stable isotopes and mass isotopomer study of fatty acid and cholesterol synthesis, A review of the MIDA approach. Advances in Experimental Medicine and Biology, 399, 95-114.

Lee, W. N., Boros, L. G., Puigjaner, J., Bassilian, S., Lim, S., \& Cascante, M. (1998). Mass isotopomer study of the nonoxidative pathways of the pentose cycle with $[1,2-13 \mathrm{C} 2]$ glucose. The American Journal of Physiology, 274, E843-E851.

Lee, M. H., Hong, I., Kim, M., et al. (2007). Gene expression profiles of murine fatty liver induced by the administration of valproic acid. Toxicology and Applied Pharmacology, 220, 45-59. doi: 10.1016/j.taap.2006.12.016.
Lee, W. N., Sorou, S., \& Bergner, E. A. (1991). Glucose isotope, carbon recycling, and gluconeogenesis using [U-13C]glucose and mass isotopomer analysis. Biochemical Medicine and Metabolic Biology, 45, 298-309. doi:10.1016/0885-4505(91)90034-I.

Lee, J. Y., Timmins, J. M., Mulya, A., et al. (2005). HDLs in apoA-I transgenic Abca1 knockout mice are remodeled normally in plasma but are hypercatabolized by the kidney. Journal of Lipid Research, 46, 2233-2245. doi:10.1194/jlr.M500179-JLR200.

Lian, X. Y., Khan, F. A., \& Stringer, J. L. (2007). Fructose-1,6bisphosphate has anticonvulsant activity in models of acute seizures in adult rats. The Journal of Neuroscience, 27, 1200712011. doi:10.1523/JNEUROSCI.3163-07.2007.

Linden, K. C., DeHaan, C. L., Zhang, Y., et al. (2006). Renal expression and localization of the facilitative glucose transporters GLUT1 and GLUT12 in animal models of hypertension and diabetic nephropathy. American Journal of Physiology. Renal Physiology, 290, F205-F213. doi:10.1152/ajprenal.00237.2004.

Lindley, D. V. (1987). Regression and correlation analysis (Vol. 4, pp. 23-120). New Palgrave: A Dictionary of Economics.

Nowak, G., \& Schnellmann, R. G. (1995). Improved culture conditions stimulate gluconeogenesis in primary cultures of renal proximal tubule cells. The American Journal of Physiology, 268, C1053-C1061.

Perucca, E. (2002). Pharmacological and therapeutic properties of valproate: A summary after 35 years of clinical experience. CNS Drugs, 16, 695-714. doi:10.2165/00023210-200216100-00004.

Powell, N., Smith, K., \& Fiander, A. (2006). Recovery of human papillomavirus nucleic acids from liquid-based cytology media. Journal of Virological Methods, 137, 58-62. doi:10.1016/j. jviromet.2006.05.033.

Puchowicz, M. A., Bederman, I. R., Comte, B., et al. (1999). Zonation of acetate labeling across the liver: Implications for studies of lipogenesis by MIDA. The American Journal of Physiology, 277, E1022-E1027.

Sarwal, R., Sanyal, S. N., \& Khera, S. (1989). Lipid metabolism in Trichuris globulosa (Nematoda). Journal of Helminthology, 63, 287-297.

Schnackenberg, L. K., Jones, R. C., Thyparambil, S., et al. (2006). An integrated study of acute effects of valproic acid in the liver using metabonomics, proteomics, and transcriptomics platforms. Omics, 10, 1-14. doi:10.1089/omi.2006.10.1.

Silva, M. F., Ijlst, L., Allers, P., et al. (2004). Valproyl-dephosphoCoA: A novel metabolite of valproate formed in vitro in rat liver mitochondria. Drug Metabolism and Disposition: The Biological Fate of Chemicals, 32, 1304-1310. doi:10.1124/dmd.

Simon, D., \& Penry, J. K. (1975). Sodium Di-N-propylacetate (DPA) in the treatment of epilepsy. Epilepsia, 16, 549-573. doi: 10.1111/j.1528-1157.1975.tb04738.x.

Slatter, T. L., Jones, G. T., Williams, M. J., van Rij, A. M., \& McCormick, S. P. (2008). Novel rare mutations and promoter haplotypes in ABCA1 contribute to low-HDL-C levels. Clinical Genetics, 73, 179-184. doi:10.1111/j.1399-0004.2007.00940.x.

Tang, W., \& Abbott, F. S. (1996). Bioactivation of a toxic metabolite of valproic acid, (E)-2-propyl-2,4-pentadienoic acid, via glucuronidation, LC/MS/MS characterization of the GSH-glucuronide diconjugates. Chemical Research in Toxicology, 9, 517-526. doi: $10.1021 / \mathrm{tx} 950120 \mathrm{y}$.

Tang, W., \& Abbott, F. S. (1997). A comparative investigation of 2propyl-4-pentenoic acid (4-ene VPA) and its alpha-fluorinated analogue: Phase II metabolism and pharmacokinetics. Drug Metabolism and Disposition: The Biological Fate of Chemicals, $25,219-227$.

Thurston, J. H., Hauhart, R. E., Schulz, D. W., Naccarato, E. F., Dodson, W. E., \& Carroll, J. E. (1981). Chronic valproate administration produces hepatic dysfunction and may delay brain maturation in infant mice. Neurology, 31, 1063-1069. 
Wong, H. Y., Chu, T. S., Lai, J. C., et al. (2005). Sodium valproate inhibits glucose transport and exacerbates Glut1-deficiency in vitro. Journal of Cellular Biochemistry, 96, 775-785. doi: $10.1002 / j \mathrm{cb} .20555$.

Xiao, G., Garg, M., Wong, D., Lim, S., Go, V. L. W., \& Lee, W. N. (2008). Determination of protein synthesis in vivo using labeling from deuterated water and analysis of MALDI-TOF spectrum. Journal of Applied Physiology, 104, 828-836.

Xu, J., Lee, W. N., Xiao, G., et al. (2003). Determination of a glucosedependent futile recycling rate constant from an intraperitoneal glucose tolerance test. Analytical Biochemistry, 315, 238-246. doi:10.1016/S0003-2697(02)00709-1. 